\title{
Cost Effectiveness of Denosumab Compared with Oral Bisphosphonates in the Treatment of Post-Menopausal Osteoporotic Women in Belgium
}

\author{
Mickaël Hiligsmann and Jean-Yves Reginster \\ Pharmacoeconomics Research Unit, University of Liège, Liège, Belgium
}

Background: Denosumab has recently been shown to be well tolerated, to increase bone mineral density (BMD) and to significantly reduce the risk of hip, vertebral and non-vertebral fractures in the FREEDOM (Fracture REduction Evaluation of Denosumab in Osteoporosis every 6 Months) trial. It is becoming increasingly important to evaluate not only the therapeutic value of a new drug but also the cost effectiveness compared with the most relevant treatment alternatives.

Objective: The objective of this study was to estimate the cost effectiveness of denosumab compared with oral bisphosphonates (branded and generic drugs) in the treatment of post-menopausal osteoporotic women in Belgium.

Methods: Cost effectiveness of 3 years of treatment with denosumab was compared with branded risedronate and branded and generic alendronate using an updated version of a previously validated Markov microsimulation model. The model was populated with relevant cost, adherence and epidemiological data for Belgium from a payer perspective and the results were presented as costs per QALY gained ( $€$, year 2009 values). Analyses were performed in populations (aged $\geq 60$ years) in which osteoporosis medications are currently reimbursed in many European countries, i.e. those with BMD $\mathrm{T}$-score of -2.5 or less or prevalent vertebral fracture. Patients receiving denosumab were assumed to have a $46 \%$ lower risk of discontinuation than those receiving oral bisphosphonates, and the effect of denosumab after treatment cessation was assumed to decline linearly to zero over a maximum of 1 year. Results: Denosumab was cost effective compared with all other therapies, assuming a willingness to pay of $€ 40000$ per QALY gained. In particular, denosumab was found to be cost effective compared with branded alendronate and risedronate at a threshold value of $€ 30000$ per QALY and denosumab was dominant (i.e. lower cost and greater effectiveness) compared with risedronate from the age of 70 years in women with a T-score of -2.5 or less and no prior fractures. The cost effectiveness of denosumab compared 
with generic alendronate was estimated at $€ 38514$, $€ 22220$ and $€ 27862$ per QALY for women aged 60, 70 and 80 years, respectively, with T-scores of -2.5 or less. The equivalent values were $€ 37167, € 19718$ and $€ 19638$ per QALY for women with prevalent vertebral fractures.

Conclusion: This study suggests, on the basis of currently available data, that denosumab is a cost-effective strategy compared with oral bisphosphonates (including generic alendronate) for the treatment of post-menopausal osteoporotic women, aged $\geq 60$ years in Belgium. Denosumab therefore appears to have the potential to become a first-line treatment for post-menopausal women with osteoporosis. However, further studies would be required to evaluate the long-term safety and adherence of denosumab in real-world clinical practice as well as head-to-head effectiveness compared with oral bisphosphonates.

\section{Key messages}

This study suggests, on the basis of currently available data, that denosumab is a cost-effective strategy compared with branded and generic bisphosphonates for the treatment of postmenopausal osteoporotic women, aged $\geq 60$ years in Belgium

Denosumab appears to have the potential to become a first-line treatment for post-menopausal women with osteoporosis

Further studies would be required to evaluate the long-term safety and adherence of denosumab in real-world clinical practice as well as head-to-head effectiveness compared with oral bisphosphonates

\section{Background}

Osteoporosis has become a major worldwide health problem, especially in countries with an aging population. It is a disease characterized by low bone mass and deterioration of bone tissue that leads to an increased risk of fracture. One in three women aged $>50$ years will experience one or more fractures attributable to osteoporosis. ${ }^{[1,2]} \mathrm{In}$ Europe alone in 2000, the number of osteoporotic fractures was estimated at 3.79 million, of which $23.5 \%$ were hip fractures. ${ }^{[3]}$ Osteoporotic fractures may lead to chronic pain, disability, a reduced quality of life (QOL) and increased mortality. Moreover, they place a heavy burden on healthcare resources, with total direct cost estimated in Europe at $€ 31.7$ billion each year (year 2000 value). ${ }^{[4]}$
Oral bisphosphonates are the first-line treatment for osteoporosis, with demonstrated efficacy in reducing the risks of vertebral and non-vertebral fractures. ${ }^{[5]}$ However, their therapeutic benefits in clinical practice are often compromised by poor adherence. $^{[6-8]}$ Approximately $75 \%$ of women who initiate oral bisphosphonates were shown to be non-adherent within 1 year and 50\% discontinued therapy by this time, ${ }^{[9]}$ resulting in significant clinical and economic burden. ${ }^{[10]}$ Determinants of poor adherence include inconvenient regimens, adverse effects and lack of motivation. ${ }^{[11,12]}$

Denosumab represents a new therapeutic opportunity for the treatment of osteoporosis. The FREEDOM (Fracture REduction Evaluation of Denosumab in Osteoporosis every six Months) trial has recently shown subcutaneous administration of $60 \mathrm{mg}$ of denosumab every 6 months to be well 
tolerated, to increase bone mineral density (BMD) and to significantly reduce the risks of vertebral, non-vertebral and hip fractures in women with osteoporosis over 3 years. ${ }^{[13]}$ Based on those findings, denosumab was granted marketing authorization from the European Medicines Agency in May $2010 .{ }^{[14]}$ In addition, patients were shown to be more satisfied and less bothered with a 6-month injection regimen for osteoporosis than with a weekly oral tablet. ${ }^{[15]}$ Less frequent dosing schedules may, in principle, contribute to improved adherence to therapy. ${ }^{[16]}$

For healthcare decision makers, it would also be important to know whether denosumab represents 'good value for money' compared with the most relevant alternative treatments. Costeffectiveness studies are increasingly utilized in pricing and reimbursement decisions..$^{[17,18]}$ Decisionanalytic models are necessary to extrapolate beyond clinical trial data and hence to capture all relevant costs and consequences. In addition, models allow the integration of efficacy and safety data from different clinical trials and thus comparison of competing treatments, an important feature since randomized controlled trials (RCTs) rarely compare active therapies.

The objective of this study was, therefore, to evaluate the cost effectiveness of denosumab compared with oral bisphosphonates (i.e. branded risedronate, branded and generic alendronate) in the treatment of post-menopausal osteoporotic women aged $\geq 60$ years in Belgium.

\section{Methods}

\section{Analysis}

Analyses were performed from the Belgian healthcare-payer perspective with a lifetime horizon. The primary outcome measure was the incremental cost-effectiveness ratio (ICER) between denosumab and comparators, expressed as incremental cost per QALY gained. In the basecase scenario, annual discount rates of $3.0 \%$ and $1.5 \%$ were used for costs and QALYs, respectively, as recommended by the Belgian Healthcare Knowledge Centre (KCE) guidelines. ${ }^{[19]}$

Cost effectiveness was assessed in women (aged $\geq 60$ years) for whom osteoporosis therapies are currently reimbursed in several European countries, i.e. those with BMD T-scores of -2.5 or less, or prevalent vertebral fractures.

Comparators were two oral bisphosphonates (alendronate and risedronate), the most widely prescribed drugs for the treatment and prevention of post-menopausal osteoporosis. Since alendronate has recently become available as a generic drug, it was chosen as a reference comparator.

\section{Model Structure}

An updated version of a recently published and validated Markov microsimulation model ${ }^{[20]}$ was used to assess the cost effectiveness of denosumab. For the purpose of this analysis, a 6-month cycle length was considered and several data values were updated, including the long-term cost following hip fracture and mortality rates. The model was programmed using the software TreeAge Pro 2009 Suite, release 1.0.2 (TreeAge Software Inc., Williamston, MA, USA).

Figure 1 provides an overview of the model. The model health states are 'no fracture', 'death', 'hip fracture', 'clinical vertebral fracture', 'wrist fracture', 'other fracture' and the corresponding post-fracture states. A new feature of the model was the introduction of post-fracture states. This revision has been required as many parameters (e.g. fracture disutility, excess mortality) were only estimated over a 1-year period. The model followed the patients individually until they died or reached the age of 105 years. The time horizon to evaluate the benefit of a particular intervention should be long enough to capture long-term impact on QOL and costs. Because fractures have a long-term impact on both, the use of a lifetime horizon is the most appropriate and has therefore been recommended for cost-effectiveness analyses of osteoporosis. ${ }^{[21]}$ A 6-month cycle length was chosen because withdrawals from denosumab can occur every 6 months.

All the women, one at a time, began in the "no fracture' state and had, every 6 months, a probability of having a fracture of the hip, clinical vertebrae, wrist or other site or dying. Women in a fracture state can stay in the same fracture state if they re-fracture, change to another fracture 


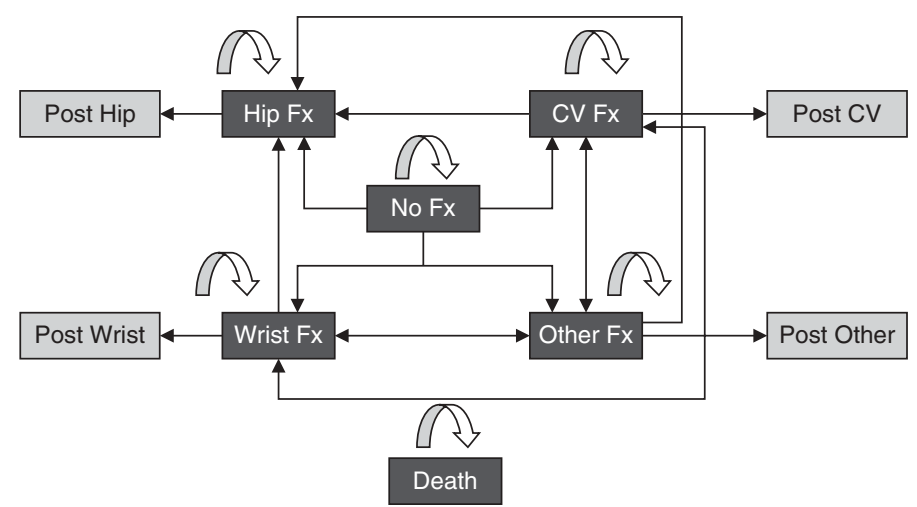

Fig. 1. Model structure. Transitions to death and from post-fracture (Fx) states to any Fx states, 'Death' and 'No Fx' were excluded from the graph for simplicity. $\mathbf{C V}=$ clinical vertebral.

state, die or change in the next cycle to the postfracture state. Because hip fracture is associated with extra costs in the year following the fracture that are greater than the hospitalization cost of any other fracture, patients who have had a hip fracture were only at risk for another hip fracture

Table I. Incidence of first fracture, cost ( $€$, year 2009 values) and utility data used in the model

\begin{tabular}{|c|c|}
\hline Parameter & Data \\
\hline \multicolumn{2}{|l|}{ Incidence (annual rate per 1000) of first fracture ${ }^{[1]}$} \\
\hline Hip fracture & $\begin{array}{l}1.57 \text { (60-64y), } 1.88 \text { (65-69y), } 4.49 \text { (70-74y), } 6.69(75-79 y) \\
12.13 \text { (80-84y), } 19.65 \text { (85-89y), } 27.29 \text { (90-94y), 31.72 (95-105y) }\end{array}$ \\
\hline Clinical vertebral fracture & $\begin{array}{l}1.93(60-64 y), 2.10(65-69 y), 5.16(70-74 y), 4.83(75-79 y) \\
5.70(80-84 y), 7.60(85-89 y), 10.55(90-94 y), 12.26(95-105 y)\end{array}$ \\
\hline Wrist fracture & $\begin{array}{l}7.05 \text { (60-64 y), } 6.07 \text { (65-69y), } 8.91 \text { (70-74y), } 6.23 \text { (75-79y), } \\
6.15 \text { (80-84y), } 6.97 \text { (85-89y), 9.68 (90-94y), } 11.26 \text { (95-105y) }\end{array}$ \\
\hline Other fracture & $\begin{array}{l}3.77(60-64 y), 5.45(65-69 y), 7.83(70-74 y), 9.74(75-79 y) \\
13.02(80-84 y), 21.09(85-89 y), 29.30(90-94 y), 34.05(95-105 y)\end{array}$ \\
\hline \multicolumn{2}{|l|}{ Direct fracture cost } \\
\hline Hip, first 6 months ${ }^{[22]}$ & $\begin{array}{l}9821(60 y), 10183(65 y), 12532(70 y), 13075(75 y), 13798(80 y) \\
12713(85 y), 12532(90-105 y)\end{array}$ \\
\hline Hip, extra cost in the year following the fracture ${ }^{[23]}$ & 7870 \\
\hline Hip, yearly long-term costs ${ }^{[22]}$ & 1102 (60-69y), 1272 (70-79y), 2544 (80-89y), 3392 (90-99y), 5088 (100-105y) \\
\hline CV, first 6 months ${ }^{[24,25]}$ & 2592 \\
\hline Wrist, first 6 months ${ }^{[26]}$ & 2302 \\
\hline Other, first 6 months ${ }^{[24,25]}$ & 3819 \\
\hline \multicolumn{2}{|l|}{ Monitoring cost ${ }^{[27]}$} \\
\hline Physician visit & 20 \\
\hline BMD measurement & 47 \\
\hline \multicolumn{2}{|l|}{ Health utility values ${ }^{[28]}$} \\
\hline General population & $0.83(60-69 y), 0.77(70-79 y), 0.72(80-105 y)$ \\
\hline Hip (first year/subsequent years) ${ }^{a}$ & $0.80 / 0.90$ \\
\hline CV (first year/subsequent years) ${ }^{a}$ & $0.72 / 0.93$ \\
\hline Wrist (first year/subsequent years) ${ }^{a}$ & $0.94 / 1.00$ \\
\hline Other (first year/subsequent years) ${ }^{a}$ & $0.91 / 1.00$ \\
\hline
\end{tabular}


Table II. Estimates of the relative risk of fracture for women with bone mineral density T-score of -2.5 or less and with prevalent vertebral fracture

\begin{tabular}{|c|c|c|c|c|c|c|c|c|}
\hline \multirow[t]{2}{*}{ Age range $(y)$} & \multicolumn{4}{|c|}{ T-score of -2.5 or less } & \multicolumn{4}{|c|}{ Prevalent vertebral fractures } \\
\hline & hip & vertebral & wrist & other & hip & vertebral & wrist & other \\
\hline $60-69$ & 3.39 & 2.18 & 1.61 & 1.90 & 2.30 & 4.40 & 1.40 & 1.80 \\
\hline $70-79$ & 2.25 & 1.77 & 1.43 & 1.61 & 2.17 & 4.06 & 1.36 & 1.72 \\
\hline $80-89$ & 1.57 & 1.51 & 1.30 & 1.42 & 2.05 & 3.75 & 1.32 & 1.65 \\
\hline $90-99$ & 1.57 & 1.51 & 1.30 & 1.42 & 1.95 & 3.48 & 1.29 & 1.58 \\
\hline $100-105$ & 1.57 & 1.51 & 1.30 & 1.42 & 1.85 & 3.23 & 1.26 & 1.52 \\
\hline
\end{tabular}

or dying in the first cycle following the fracture. Women in any post-fracture states might have a new fracture (all fracture types are again possible), die or move to the 'no fracture' state.

Patient history, i.e. prior fractures and residential status (living either in the community or in a nursing home), was recorded by tracker variables and used in calculations of transition probabilities, costs and utilities to reflect the long-term effects of fracture and residential status of patients. Using tracker variables, the restrictive assumptions regarding patient movements to health states used in cohort models are not needed. ${ }^{[20]}$ A patient can therefore go back to the 'no fracture' state, even after a hip or clinical vertebral fracture. For an illustration on how the model integrates memory, please refer to Hiligsmann et al. ${ }^{[20]}$

\section{Model Inputs}

\section{Fracture Incidence}

Yearly probabilities of first fracture (at specific sites) in the general population were taken from a recently published source ${ }^{[1]}$ (see table I). Since the incidence of non-hip fractures was not known in Belgium, this study assumed that the age-specific ratio of index fracture to hip fracture in Belgium was the same as found in other countries. This assumption, frequently used in costeffectiveness modelling of osteoporosis, appears to hold true for West European countries, the US and Australia. ${ }^{[29]}$

Fracture risk in the general population needs to be adjusted to accurately reflect the fracture risk in the target populations (table II). Low BMD and prior fractures are associated with increased fracture risk. ${ }^{[30,31]}$ For women with a
BMD T-score of -2.5 or less, the relative risks (RRs) were calculated using a method previously described, ${ }^{[32]}$ which estimates the risk of individuals below the threshold value compared with the fracture risk in the general population of that age. The number of standard deviations of BMD below the age-matched average BMD was derived from the US National Health and Nutrition Examination Survey (NHANES) III ${ }^{[33]}$ database, for which young adult BMD values were not significantly different from Belgian estimates (suggesting that values in older adults may also be similar between the two populations). ${ }^{[34]}$ One standard deviation decrease in BMD was associated with an increase in age-adjusted RR $(95 \%$ CI) of $1.8(1.1,1.7), 1.4(1.4,1.6)$ and $1.6(1.4,1.8)$ for clinical vertebral, wrist and other osteoporotic fracture, respectively. ${ }^{[31]}$ The age-adjusted RR for hip fracture ranged from $3.1(2.4,3.9)$ at 60 years to $1.9(1.8,2.1)$ at 85 years. ${ }^{[35]}$ For women with prevalent vertebral fractures, the RRs were $2.3(2.0,2.8), 4.4(3.6,5.4), 1.4(1.2,1.7)$ and 1.8 $(1.7,1.9)$ for hip, clinical vertebral, wrist and other fracture, respectively. ${ }^{[30]}$ These RRs were reduced by $10 \%$ per decade above the age of 70 years. ${ }^{[36]}$ For these women, the BMD was assumed to be the same as the age-matched NHANES population. However, patients with a prior fracture are likely to have lower BMD than the general population and the cost effectiveness of denosumab may therefore be underestimated in this group.

In the group of women with BMD T-scores of -2.5 or less and no prior fracture, fracture risk was also adjusted when a new fracture occurred during the simulation. An increased risk of subsequent fractures was modelled for women who had a prior fracture at the same location. These 
increased RRs $(95 \% \mathrm{CI})$ were $4.4(3.6,5.4), 2.3$ $(1.5,3.7), 3.3(2.0,5.3)$ and $1.9(1.7,2.2)$ for vertebral, hip, wrist and other fractures, respectively. ${ }^{[30]}$ As the underlying risk of first fracture may contain prior fracture at other sites and a multiplicative hypothesis could not be supported at this time, we conservatively did not model an increased risk of subsequent fractures at sites different from that of the prior fractures. However, an increased RR of 2.3 (95\% CI 2.0, 2.8) is modelled for a hip fracture after a vertebral fracture, because this effect is largely supported by the literature. ${ }^{[30]}$ All these increased RRs were increased by a factor of 1.7 during the year following the fracture $^{[37]}$ and were reduced by $10 \%$ per decade above the age of 70 years. ${ }^{[36]}$ Further subsequent fractures of the same type were assumed to have no additional effect. In the group of women with prevalent vertebral fractures, fractures during the simulation process were not associated with an additional increase in fracture risk.

\section{Mortality Rates}

Age-specific mortality rates were derived from the Belgian National Institute of Statistics. ${ }^{[38]}$ Excess mortality following a hip fracture was derived from a Swedish-based population study. ${ }^{[39]}$ This study gave detailed and conservative estimates in comparison with other studies. ${ }^{[40]}$ The excess mortality was assumed to be of similar magnitude following a clinical vertebral fracture, ${ }^{[40-43]}$ and it decreased in subsequent years for both types of fracture. Because excess mortality may be attributable to co-morbidities, we conservatively assumed that only $25 \%$ of the excess mortality following a hip or vertebral fracture could be directly or indirectly attributable to the fractures themselves. ${ }^{[42,43]}$

Therefore, the relative mortality rate in the year following a hip or clinical vertebral fracture was estimated at 2.74, 2.09, 1.54, 1.22 and 1.13 for women aged 60, 70, 80, 90 and 100 years, respectively. In subsequent years, the corresponding values were $1.66,1.43,1.16,1.01$ and 1.00 , respectively. We also assumed that non-hip, nonvertebral fractures were not associated with an increased mortality and in a conservative manner that a second and further fracture at the same site will cause no greater mortality excess, except the increase for the year after they occur.

\section{Utilities}

The QALY is an attractive outcome measure in osteoporosis because therapies have consequences on both length and quality of life. In the absence of data specific to Belgium, the utility values used in the model were derived from a systematic review of the literature. ${ }^{[28]}$ Utility values in the general population as well as relative reductions due to fractures in the year following the fracture and in subsequent years are reported in table I. Reference values for fracture disutility were generally derived from a Swedish study, in which health-related QOL data were collected with the EQ-5D questionnaire at baseline, 4 months and 12 months after different fractures from a patient sample of 635 male and female patients.

When a second fracture occurred at the same site, the disutility applied to the first fracture event was reduced by $50 \% .{ }^{[20]}$ This assumption is supported by recent studies showing that the number of fractures is a significant determinant of QOL. ${ }^{[44-46]}$

\section{Cost Data}

The healthcare payer perspective was adopted for all cost estimates. Only direct medical costs were considered. All costs were expressed in $€$, year 2009 values, using the healthcare product price index to adjust to year 2009 values. ${ }^{[47]}$

The costs of hip fracture were retrieved from two published studies conducted in Belgium: Reginster et al. ${ }^{[22]}$ estimated the hospitalization cost of hip fracture based on a retrospective analysis of 2374 patients, while Autier et al. ${ }^{[23]}$ estimated the extra costs during the year following the hospitalization from a prospective controlled study including 159 women. Total direct costs in the year following a hip fracture were therefore estimated to be between $€ 17691$ and $€ 21668$, according to age.

Wrist fracture cost was estimated at €2302. ${ }^{[26]}$ The cost of clinical vertebral and other fractures has never been estimated in Belgium and these were quantified relative to hip fracture cost. Assuming that these represent $17 \%$ and $25 \%$, respectively, of hip fracture cost, ${ }^{[24,25]}$ they were estimated, respectively, at $€ 2592$ and at $€ 3819$ 
(unlike Autier et al., ${ }^{[23]}$ nursing home costs were not integrated for these proportion factors, so we reduced hip fracture costs by $50 \%$ of their extra costs before applying the proportion factors).

Hip fractures are also associated with longterm costs. Hip fracture costs for the second and subsequent years were based on the proportion of patients being institutionalized following the fracture, ranging from 5\% (for the age range 50-60 years) to $30 \%$ (for women aged $\geq 90$ years). ${ }^{[22]}$ Because women might be institutionalized later in life, regardless of their hip fracture, an adjustment was made to only include long-term costs attributable to the fracture itself. ${ }^{[20]}$ Non-hip fractures were conservatively assumed to be not associated with long-term costs.

\section{Interventions}

In order to assess the cost effectiveness of osteoporosis medications, data were required on fracture reduction efficacy (at specific sites), treatment effect duration, the effect of treatment after stopping therapy, adherence to therapy, adverse events and intervention cost. ${ }^{[20]}$

The effect of denosumab on fracture risk was derived from the 3 -year results of the FREEDOM trial. ${ }^{[13]}$ As compared with placebo, denosumab reduced the risk of hip fracture by $40 \%$ (RR 0.60 , $95 \%$ CI $0.37,0.97)$, the risk of clinical vertebral fracture by $69 \%$ (RR $0.31,95 \%$ CI $0.20,0.47$ ) and the risk of other fracture (including wrist fracture) by $20 \%$ (RR $0.80,95 \%$ CI $0.67,0.95$ ) using the estimated fracture risk reduction for non-vertebral fractures. The effect of oral bisphosphonates was derived from a recent meta-analysis conducted for the UK National Institute for Health and Clinical Excellence appraisal, which included large RCTs involving post-menopausal women. ${ }^{[48]}$ The RRs of fracture are reported in table III. The same efficacy was assumed for generic and branded alendronate.

Patients in the model received treatment for a maximum of 3 years, as in the clinical trials. However, the model simulated a woman's lifetime in order to capture all costs and consequences of fractures experienced during the treatment period. After stopping therapy, the effect of oral bisphosphonates on fracture risk was assumed to decline linearly to zero for a period (called offset time) similar to the duration of therapy, in line with clinical studies ${ }^{[52,53]}$ and previous cost-effectiveness analyses. ${ }^{[54]}$ For denosumab, we suggested a declin-

Table III. Effectiveness and cost data for denosumab, alendronate and risedronate

\begin{tabular}{|c|c|c|c|}
\hline & Denosumab & Alendronate & Risedronate \\
\hline \multicolumn{4}{|c|}{ Relative risk of fracture during therapy $(95 \% \mathrm{Cl})^{[13,48]}$} \\
\hline hip & $0.60(0.37,0.97)$ & $0.62(0.40,0.96)$ & $0.74(0.59,0.93)$ \\
\hline clinical vertebral & $0.31(0.20,0.47)$ & $0.56(0.46,0.67)$ & $0.64(0.52,0.78)$ \\
\hline wrist & $0.80(0.67,0.95)$ & $0.85(0.67,1.09)$ & $0.68(0.43,1.07)$ \\
\hline other fractures & $0.80(0.67,0.95)$ & $0.82(0.73,0.93)$ & $0.80(0.72,0.90)$ \\
\hline Offset time ${ }^{a}$ & Maximum 1 y & Equal to therapy duration & Equal to therapy duration \\
\hline \multicolumn{4}{|l|}{ Adherence to therapy } \\
\hline $\begin{array}{l}\text { probabilities }(\%) \text { of } \\
\text { discontinuing therapy }{ }^{[49]}\end{array}$ & $\begin{array}{l}22.9(6 \mathrm{mo}), 9.8(1 \mathrm{y}), 4.5(1.5 \mathrm{y}) \\
3.0(2 \mathrm{y}) \text { and } 2.2(2.5 \mathrm{y})\end{array}$ & $\begin{array}{l}42.5(6 \mathrm{mo}), 18.1(1 \mathrm{y}), 8.3(1.5 \mathrm{y}) \\
5.6(2 \mathrm{y}) \text { and } 4.1(2.5 \mathrm{y})\end{array}$ & $\begin{array}{l}42.5(6 \mathrm{mo}), 18.1(1 \mathrm{y}), 8.3(1.5 \mathrm{y}) \\
5.6(2 \mathrm{y}) \text { and } 4.1(2.5 \mathrm{y})\end{array}$ \\
\hline $\begin{array}{l}\text { probabilities }(\%) \text { of } \\
\text { poor compliance } \\
(\mathrm{MPR}<80 \%)^{[49]}\end{array}$ & 0 & $\begin{array}{l}\text { First y: } 23.9 \\
\text { Second y: } 4.0 \\
\text { Third y: } 1.2\end{array}$ & $\begin{array}{l}\text { First y: } 23.9 \\
\text { Second y: } 4.0 \\
\text { Third y: } 1.2\end{array}$ \\
\hline $\begin{array}{l}\text { increased risk } \\
\text { associated with } \\
\text { poor compliance } \\
{[\mathrm{RR}(95 \% \mathrm{Cl})]}\end{array}$ & NA & $\begin{array}{l}\text { Hip fracture: } 1.35(1.17,1.56)^{[49]} \\
\text { All non-hip fractures: } 1.17 \\
(1.09,1.25)^{[50]}\end{array}$ & $\begin{array}{l}\text { Hip fracture: } 1.35(1.17,1.56)^{[49]} \\
\text { All non-hip fractures: } 1.17 \\
(1.09,1.25)^{[50]}\end{array}$ \\
\hline Annual drug cost $(€)^{[51]}$ & 414.9 & $\begin{array}{l}\text { Branded: } 283.0 \\
\text { Generic: } 159.8\end{array}$ & 414.9 \\
\hline
\end{tabular}

a Effect of treatment after stopping therapy; duration of the linear decrease in fracture risk reduction.

$\mathbf{M P R}=$ medication possession ratio; $\mathbf{N A}=$ not applicable; $\mathbf{R} \mathbf{R}=$ relative risk . 
ing residual effect for a maximum 1-year period, as previously assumed. ${ }^{[55]}$ This assumption is rather conservative since recent data have shown that the treatment benefit achieved (changes in BMD) with 2 years of denosumab therapy was reversed within 2 years of treatment discontinuation. ${ }^{[56]}$

\section{Adherence}

Adherence to osteoporosis medications is a major concern $^{[57]}$ and may substantially affect costeffectiveness results. ${ }^{[58,59]}$ For modelling purposes, adherence was divided into 'compliance' (i.e. "the extent to which a patient acts in accordance with the prescribed interval and dose of a dosing regimen"'[60]) and 'persistence' (i.e. "the duration of time from initiation to discontinuation of therapy"[60]). Data on adherence to oral bisphosphonates were obtained from the database used by Rabenda et al.,${ }^{[49]}$ who conducted a retrospective cohort analysis including pharmacy records of post-menopausal women who had received a first prescription of oral bisphosphonates (daily and weekly combined) in Belgium between April 2001 and June 2004. Compliance was quantified as the number of doses taken divided by the number of doses prescribed - the medication possession ratio (MPR)-and medication persistence was captured by the proportion of patients still receiving medication at different time periods. A refill gap of 5 weeks was used to assess persistence; i.e. persistence was calculated as the number of days from the initial prescription to a gap of more than 5 weeks after completion of the previous refill. The methodology used to incorporate compliance and persistence with oral bisphosphonates has been published recently. ${ }^{[10,61]}$

Based on the Belgian observational study, ${ }^{[49]}$ $42.5 \%$ of women discontinued oral bisphosphonates within 6 months. For these women, no treatment effect was received ${ }^{[62]}$ and we assigned 3 months of therapy cost, as previously suggested. ${ }^{[59]}$ Another $18.1 \%, 8.3 \%, 5.6 \%$ and $4.1 \%$ of women discontinued therapy at 1 year, 1.5 years, 2 years and 2.5 years, respectively. Therefore, only $21.4 \%$ of women received 3 years of treatment. It was assumed that, if patients discontinued therapy, they received no further treatment and their offset time was similar to the duration receiving therapy.
Persistent women were considered to be compliant if their MPR was at least $80 \%$ in any given year and poorly compliant, otherwise. An MPR $>80 \%$ was commonly used to define high compliance. ${ }^{[8]}$ In the subgroup of persistent women (including 19357, 6296 and 1133 women in the first, second and third year of therapy, respectively), the probabilities of being poorly compliant (i.e. MPR <80\%) were estimated at $23.9 \%, 4.0 \%$ and $1.2 \%{ }^{[49]}$ These women experience a lower treatment efficacy. Poor compliance (MPR <80\%) was associated with a $35 \%$ increase in hip fracture rate (RR 1.35, 95\% CI 1.17, 1.56). ${ }^{[49]}$ This estimate was derived from the case-control Belgian study including 901 women who incurred a hip fracture and were persistent with oral bisphosphonates and 4505 randomly selected, matched controls. ${ }^{[49]}$ Because the Belgian study did not assess the relationship between compliance and non-hip fractures, we assumed a conservative ${ }^{[8]}$ $17 \%$ increase in other fracture rates (RR $1.17,95 \%$ CI $1.09,1.25)^{[50]}$ for poorly compliant women. The RRs from the systematic review were applicable to the population with compliance of $\geq 80 \%$. For instance, if alendronate was assumed to reduce the risk of hip fracture by $38 \%$, then compliant women would experience a $38 \%$ reduction in hip fracture while poorly compliant women would experience only a $16.3 \%(0.62 \times 1.35=0.84)$ reduction in hip fracture. For poorly compliant women, drug cost was restricted to the average MPR in this group, estimated at $74 \%, 75 \%$ and $78 \%$ in the first, second and third year of treatment, respectively.

Persistence with denosumab in real-life settings is unknown. However, it has been shown that the mode and schedule of administration may affect persistence and compliance with therapy and, in particular, less frequent regimens have been associated with better adherence. ${ }^{[16,63]}$ Therefore, persistence rates for twice-yearly injections of denosumab would be higher than those previously reported for daily or weekly bisphosphonates. This has been recently confirmed by an open-label study of 250 osteoporotic women who were randomized to either denosumab or branded oral alendronate. ${ }^{[64]}$ In the first 12 months, significantly more patients who received denosumab than alendronate were treatment persistent (risk ratio for discontinua- 
tion $0.54 ; \mathrm{p}=0.049) .{ }^{[64]}$ Patients receiving denosumab were therefore assumed in our model to have a $46 \%$ lower risk of treatment discontinuation than patients receiving oral bisphosphonates. Therefore, $22.9 \%, 9.8 \%, 4.5 \%, 3.0 \%$ and $2.2 \%$ of patients treated with denosumab were assumed to discontinue therapy at 6 months, 1 year, 1.5 years, 2 years and 2.5 years, respectively. Therefore, $57.6 \%$ of patients received 3 years of treatment. In the case of discontinuation with denosumab after 6 months, fracture efficacy was assumed during this period given the large gains in BMD previously reported ${ }^{[65,66]}$ and a declining effect over the subsequent 6 months. We also assumed, for both treatments, that if patients discontinued therapy, they received no further treatment.

\section{Intervention Costs}

Drug prices were based on the official listings of the Belgian Centre for Pharmacotherapeutic Information as at April 2010. ${ }^{[51]}$ The annual cost of risedronate and branded alendronate was estimated respectively at $€ 414.9$ (Actonel $^{\circledR}$, $€ 95.48$ for a package of 84 tablets [ $5 \mathrm{mg}$ ], once daily) and at $€ 283.0$ (Fosamax $^{\circledR}, € 65.13$ for a package of 12 [70 mg] tablets, once per week). For generic alendronate, we selected the mean cost between the lowest and the highest price of drugs, i.e. $€ 159.8$ per year (generic prices between $€ 35.67$ and $€ 37.9$ for a package of $12[70 \mathrm{mg}$ ] tablets, once per week). At the time of the analysis, denosumab was not commercially available in Belgium and for the purposes of this analysis we assumed the price of denosumab to be the same as branded risedronate (i.e. $€ 414.9$ per year). A higher price was tested in sensitivity analyses. We also assigned for both denosumab and bisphosphonate recipients the cost of two physician visits ( $€ 20$ per visit) per year of treatment and the cost of one bone density measurement (estimated at $€ 47)$ at years 1 and 3.[27]

\section{Adverse Events}

Adverse events associated with osteoporosis medications, including denosumab, are usually mild and transient. ${ }^{[5,13]}$ The cost and QOL impact of adverse events would be minor and they were therefore not included in the analysis.

\section{Sensitivity Analyses}

A series of sensitivity analyses were performed on the main model inputs and assumptions to explore the robustness of the model and the degree of impact on the results. One-way sensitivity analysis was conducted on key model parameters for a plausible range of values in order to identify the drivers of the model results and test the impact on model outcome. The probabilistic sensitivity analysis aimed to evaluate and characterize uncertainty around the model.

To perform this probabilistic analysis, a specific distribution was attributed to each parameter around the point estimate used in the base-case analysis. Log-Normal distributions were assumed for fracture risk reduction with therapy and for increased risk related to poor compliance, as recommended for RR parameters. ${ }^{[17]}$ A Beta distribution was assumed for utilities based on confidence intervals and Normal distributions, with an SD assumed to be $15 \%$ of the mean, were used for the fracture cost and fracture incidence variables given that a standard error was not available for these parameters. The distributions of the relative fracture risks associated with a BMD $\mathrm{T}$-score of -2.5 or less and with prevalent vertebral fracture were respectively assumed to be log-Normal and Uniform.

First-order Monte Carlo simulations were performed to estimate the cost effectiveness of denosumab. Base-case analysis and one-way sensitivity analyses were based on ten simulations of 200000 trials (patients) in each treatment arm (see the Model Validation section for justifications). Mean ICER and 95\% CI were calculated for each simulation. For probabilistic sensitivity analyses, the model was run 200 times based on runs of 50000 patients per treatment arm. Results are presented in the form of cost-effectiveness acceptability curves that show the probability of being cost effective as a function of the threshold willingness to pay (WTP) per QALY.

\section{Results}

\section{Model Validation}

The model performed well during validation, producing fracture incidence and mortality rates 


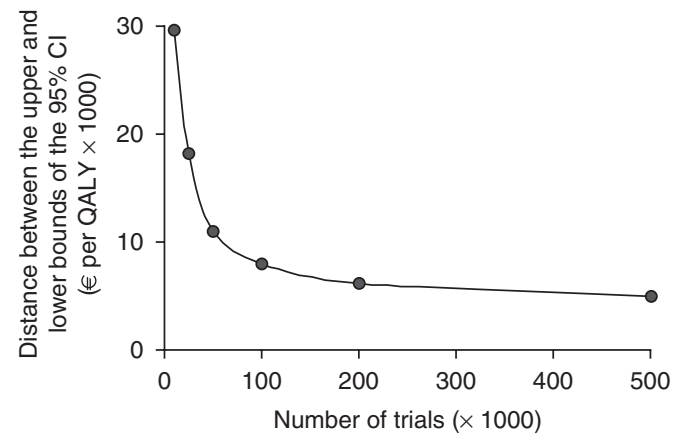

Fig. 2. Model validation: distance between the upper and lower bounds of the $95 \% \mathrm{Cl}$ of the cost effectiveness of denosumab compared with generic alendronate for a varying number of trials run ten times (in women aged 70 years with bone mineral density $\mathrm{T}$-score -2.5 or less).

that were similar to the data. Absolute lifetime risks of hip fracture and of any osteoporotic fracture were estimated, respectively, at $18.1 \%$ and $48.5 \%$ for women aged 60 years, in line with epidemiological data. ${ }^{[1]}$ Furthermore, results were very similar to those previously estimated with the first version of the model, using a 1-year cycle length. ${ }^{[20]}$ Tests on model parameters and modelling assumptions were also consistent with expected conclusions.

To determine the number of simulations, a varying number of trials $(10000-500000)$ were run ten times in women aged 70 years with BMD $\mathrm{T}$-scores of -2.5 or less, providing estimates of the cost effectiveness of denosumab compared with generic alendronate. The distance between the upper and lower bounds of the 95\% CIs reached a plateau from 200000 trials (figure 2). This number was deemed sufficient to provide stable results and was therefore selected for the analyses. Given the large computational burden when conducting probabilistic sensitivity analyses, the number of trials was restricted to 50000 for this purpose.

\section{Base-Case Analysis}

Lifetime costs, QALYs, number of fractures and the incremental cost effectiveness of denosumab compared with oral bisphosphonates for osteoporotic women aged 70 years are shown in table IV. Denosumab was more effective and generally more costly than alternative treatments. The cost per QALY gained of denosumab, lower than $€ 25000$ in all scenarios, increased when compared with a generic drug and was the lowest when compared with risedronate. In women with a BMD T-score of -2.5 or less, denosumab was also less costly than risedronate, and therefore is considered a dominant strategy. There were no other marked differences between the two target populations.

Figure 3 shows that the cost effectiveness of denosumab generally improved with increasing age. Denosumab was cost effective, compared with all other therapies, at an ICER threshold value of $€ 40000$ per QALY gained over the entire age range (60-80 years). In particular, denosumab was

Table IV. Lifetime costs ( $€$, year 2009 values), QALYs, number of fractures and incremental cost-effectiveness ratio (ICER) of denosumab compared with oral bisphosphonates in osteoporotic women aged 70 years

\begin{tabular}{|c|c|c|c|c|}
\hline Lifetime outcomes (per pt) & Denosumab & Branded alendronate & Generic alendronate & Risedronate \\
\hline \multicolumn{5}{|c|}{ Women with BMD T-score of -2.5 or less } \\
\hline Costs & 12561 & 12312 & 12169 & 12565 \\
\hline QALYS & 10.4424 & 10.4248 & 10.4248 & 10.4202 \\
\hline No. of fractures & 1.2122 & 1.2393 & 1.2393 & 1.2386 \\
\hline ICER $^{a}(95 \% \mathrm{Cl})$ & & $14120(10109,16765)$ & $22220(18628,24780)$ & $-209(-4003,1681)$ \\
\hline \multicolumn{5}{|c|}{ Women with prevalent vertebral fracture } \\
\hline Costs & 11563 & 11198 & 11055 & 11426 \\
\hline QALYS & 10.3739 & 10.3482 & 10.3482 & 10.3431 \\
\hline No. of fractures & 1.2285 & 1.2558 & 1.2558 & 1.2563 \\
\hline $\operatorname{ICER}(95 \% \mathrm{Cl})$ & & $14166(12251,16758)$ & $19718(17940,22346)$ & $4456(2573,6540)$ \\
\hline
\end{tabular}

a $€$ per QALY gained for denosumab vs each oral bisphosphonate.

$\mathbf{B M D}=$ bone mineral density; $\mathbf{p t}=$ patient. 


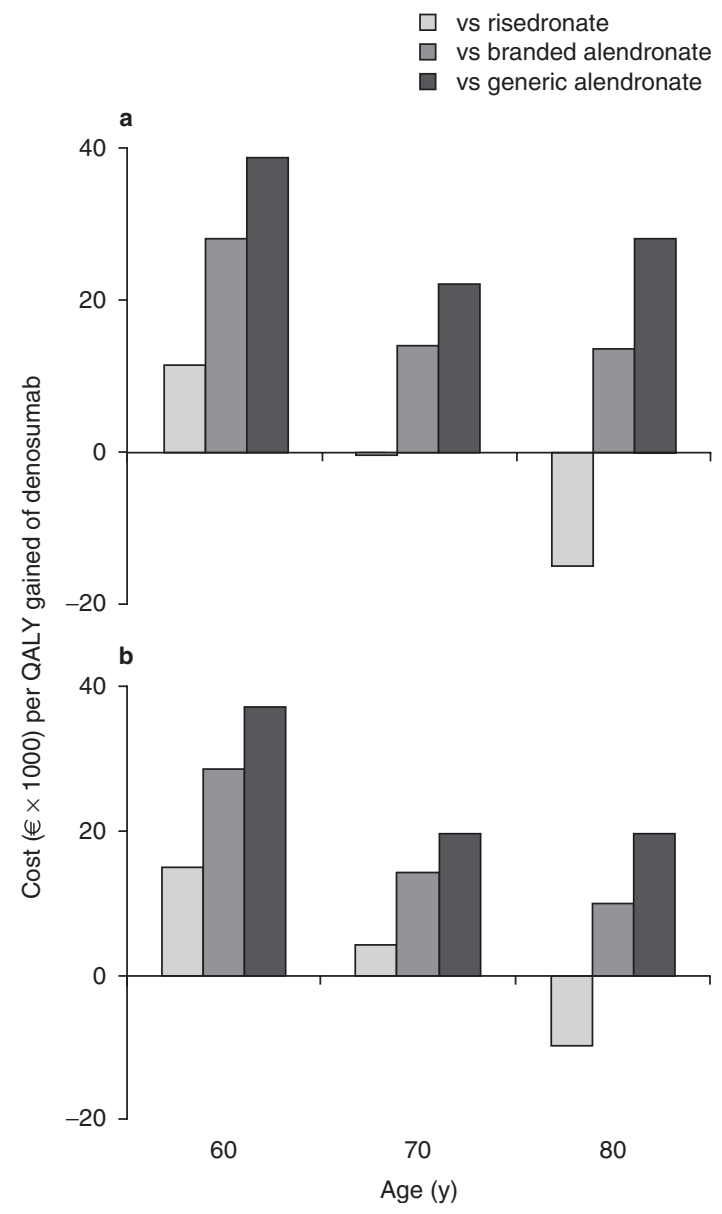

Fig. 3. Incremental cost-effectiveness ratio of denosumab compared with oral bisphosphonates according to age in women (a) with bone mineral density T-score of -2.5 or less; and (b) with prevalent vertebral fracture.

found to be cost effective compared with branded alendronate and risedronate at a threshold value of $€ 30000$ for a QALY, and denosumab was dominant compared with risedronate from the age of 70 years in women with densitometric osteoporosis. The cost effectiveness $(95 \% \mathrm{CI})$ of denosumab compared with generic alendronate was estimated at $€ 38514$ (31 664, 45 872), €22 220 (18 628, $24780)$ and $€ 27862(22685,36065)$ per QALY for women with BMD T-scores of -2.5 or less aged 60,70 and 80 years, respectively. The equivalent values were $€ 37167$ (32 506, 44 370), €19 718 (17940, 22 346) and €19638 (16936, 24144$)$ per QALY for women with prevalent vertebral fractures.

\section{One-Way Sensitivity Analyses}

Results of the one-way sensitivity analyses when comparing denosumab with generic alendronate are reported in table $\mathrm{V}$ and figure 4 . The analyses presented in table $\mathrm{V}$ suggested that the results were most sensitive to changes in fracture risk and discount rates, and less sensitive to changes in fracture cost and fracture disutility. So, the ICERs of denosumab increased by $30-41 \%$ for higher discount rates ( $5 \%$ costs, $5 \%$ benefits), and by $39-63 \%$ when fracture risk was reduced by $25 \%$. Assuming no excess mortality following hip and vertebral fracture resulted in higher cost-effectiveness ratios.

Sensitivity analyses on treatment parameters are presented in figure 4 . Results were strongly influenced by adherence to oral bisphosphonates. Reducing the probabilities of poor compliance and of discontinuing generic alendronate by $25 \%$, the ICER of denosumab was just above $€ 40000$ per QALY gained. The one-way sensitivity analysis on compliance alone did not have a large impact on the results. Results were also insensitive to the presence of monitoring costs and to therapy costs for women who discontinued oral bisphosphonates early in the course of treatment. A scenario whereby a maximum 2-year offset time was assumed for denosumab had a large impact on the results of the model. If we assumed a 2-year offset time for both interventions, the ICER decreased to $€ 12986$ (11 191, 15 267). Increasing the cost of denosumab by $15 \%$ resulted in higher ICERs, but these were still below a threshold of $€ 30000$ per QALY. Even if the cost of generic alendronate was reduced by $50 \%$, denosumab remained cost effective at $€ 26101(22533,29638)$ per QALY.

\section{Probabilistic Sensitivity Analyses}

Cost-effectiveness acceptability curves are shown in figure 5. The curves indicate the probability that the intervention is cost effective at different WTP thresholds per 1 QALY. At a threshold of $€ 40000$ per QALY, denosumab, generic alendronate and risedronate were, respectively, cost effective in $74.5 \%, 25.0 \%$ and $0.5 \%$ of simulations 
in women with BMD T-scores of -2.5 or less. The corresponding probabilities were $89.0 \%, 11.0 \%$ and $0.0 \%$ in women with prevalent vertebral fracture.

The intervention with the highest probability is the one that should be selected for treatment. Accordingly, in women with BMD T-scores of -2.5 or less, generic alendronate should be selected if WTP is less than $€ 20341$ per QALY; if it is higher, denosumab is the strategy of choice. In women with prevalent vertebral fracture, denosumab is the preferred option for a WTP higher than $€ 20333$. Risedronate was never a preferred strategy in our simulations.

\section{Discussion}

In this study, a Markov microsimulation model from a Belgian payer perspective and a lifetime horizon was used to estimate the cost effectiveness of denosumab compared with oral bisphosphonates. The KCE guidelines do not mention thresholds for cost effectiveness; ${ }^{[67]}$ however, the cost per QALY gained of denosumab was below commonly accepted thresholds of $€ 35000$ $45000 .{ }^{[54,68,69]}$ Considering these thresholds, the introduction of denosumab against oral bisphosphonates appears to be a cost-effective alternative for the treatment of post-menopausal osteoporotic women aged $\geq 60$ years in Belgium. The cost effectiveness was also robust for most of the parameters varied in sensitivity analyses. Discount rates, fracture risk and patient's adherence to oral bisphosphonates were found to be particularly influential when varied within the model.

This study provides the first pharmacoeconomic evaluation of denosumab compared with alternative treatments. We previously showed that denosumab was cost effective for post-menopausal women with low BMD, but the analysis was restricted to a comparison with no treatment. ${ }^{[55]}$ Health economic evaluations should ideally compare a new intervention with those interventions that will likely be replaced. Given that no direct comparisons are currently available, an indirect comparison was performed in this study using currently available clinical data. However, indirect comparison of efficacy between drugs may be less robust because of different baseline characteristics of the populations studied and overlapping confidence intervals for the effect of treatment. ${ }^{[70]}$ Our findings would therefore require confirmation, ideally with head-to-head observational studies of denosumab compared with oral bisphosphonates, to provide more robust data.

Analyses should also be updated as soon as new evidence becomes available. Denosumab is not currently commercially available and has no defined price. To evaluate the impact of drug price

Table V. One-way sensitivity analyses (conducted on discount rates, fracture risk, cost and disutility and mortality excess) for the cost effectiveness $^{\mathrm{a}}$ of denosumab compared with generic alendronate in osteoporotic women aged 70 years

\begin{tabular}{lll}
\hline & BMD T-score of -2.5 or less & Prevalent vertebral fractures \\
\hline Base-case analysis & $22220(18628,2478)$ & $19718(17940,22346)$ \\
Discount rates 0.00 & $17145(13261,21630)$ & $17399(15150,20411)$ \\
Discount rates 0.03 & $25850(21906,29627)$ & $22307(20397,25172)$ \\
Discount rates 0.05 & $31359(25725,38247)$ & $25681(23508,28736)$ \\
Fracture risk 25\% lower & $36125(30669,46076)$ & $27406(21739,32065)$ \\
Fracture risk 25\% higher & $12374(9186,16009)$ & $13614(11198,15584)$ \\
Fracture costs 25\% lower & $28380(24972,34676)$ & $21134(18495,23339)$ \\
Fracture costs 25\% higher & $19609(16167,24510)$ & $15899(11433,18920)$ \\
Fracture disutility 25\% lower & $27420(22634,34003)$ & $22929(21058,26086)$ \\
Fracture disutility 25\% higher & $19288(15843,21601)$ & $15577(13228,18295)$ \\
No excess mortality after hip and vertebral fractures & $34120(26331,40436)$ & $28995(25499,32421)$ \\
No excess mortality after vertebral fracture & $24807(19345,30298)$ & $25263(21157,29122)$ \\
\hline
\end{tabular}

a Cost effectiveness is expressed in cost ( $€$, year 2009 values) per QALY gained $(95 \% \mathrm{Cl})$.

BMD = bone mineral density 
Base case: $€ 22220$ per QALY

2 y offset time for denosumab and GA

2 y offset time for denosumab

Denosumab cost $15 \%$ lower

No monitoring costs

Lower GA cost for discontinuing patients ${ }^{1}$

Full compliance for $\mathrm{GA}$

GA cost $50 \%$ lower

Denosumab cost $15 \%$ higher

Denosumab cost $30 \%$ higher

GA adherence $25 \%$ higher $^{2}$

Denosumab cost $50 \%$ higher

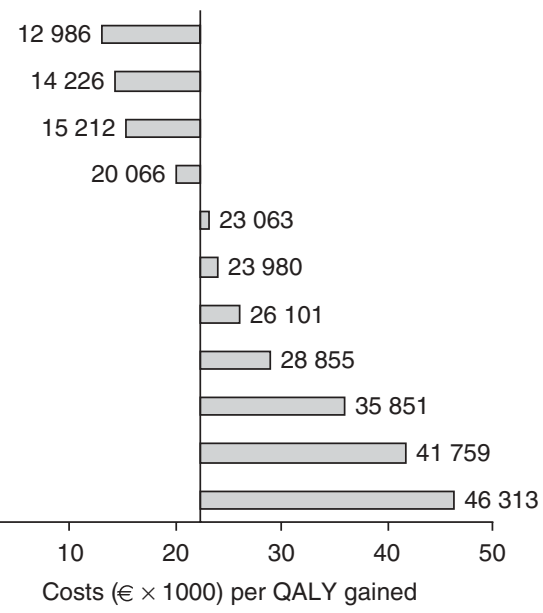

Fig. 4. Tornado diagram for one-way sensitivity analyses (conducted on treatment parameters) on the cost effectiveness of denosumab compared with generic alendronate (GA) in women aged 70 years with bone mineral density T-score of -2.5 or less. 1 Cost of 4 weeks' GA for patients who discontinue therapy within 6 months. 2 Probabilities of being poorly compliant and discontinuation rates in the real-world setting were reduced by $25 \%$.

on the results, a higher price was tested, which could affect cost effectiveness of denosumab. Additional data are also required on the longterm safety and efficacy of denosumab, the offset effect of denosumab after discontinuation and the persistence with denosumab in real-life settings. An extension of the FREEDOM trial is currently ongoing to evaluate the long-term safety and efficacy of denosumab for the treatment of post-menopausal osteoporosis. Longitudinal observational studies will also be useful to estimate the effectiveness of the drug use in current practice settings and to document real-world persistence.

Further data on generic alendronate are also needed. In this study, we assumed the same efficacy and adherence for branded and generic alendronate, although there is currently no evidence to support this assumption. Recent studies have even suggested that persistence and effectiveness with generic alendronate is poorer than with branded alendronate. ${ }^{[71,72]}$ Our comparison with generic alendronate may therefore be conservative for denosumab, by overestimating the ICER (i.e. denosumab may be more cost effective if persistence is poorer with generic alendronate), especially since we adopted a conservative approach for the effect of treatment after stopping denosumab. In addition, we have not included adverse effects with oral bisphosphonates. Although oral bisphosphonates are generally well tolerated, they are likely to be associated with upper gastrointestinal adverse events, ${ }^{[73]}$ which may require additional costs.

As with any model, there are potential limitations that should be noted. The most important are availability of data. Although data used to construct the model were based on Belgian literature whenever possible, some data were derived from other countries. In particular, the incidence of non-hip fractures and the cost of vertebral and other fractures were quantified relative to hip fracture. This proportional assumption appears to hold true for West European countries, the US and Australia. ${ }^{[24,25,29]}$ It could also be argued that hip fractures have been the fracture type considered to be the key driver of cost effectiveness of osteoporosis medications, ${ }^{[74]}$ and were estimated from Belgian published studies. Adherence to oral bisphosphonates was also derived from a local study. It would be of considerable value to update or collect additional local data (e.g. utilities, non-hip fractures). 


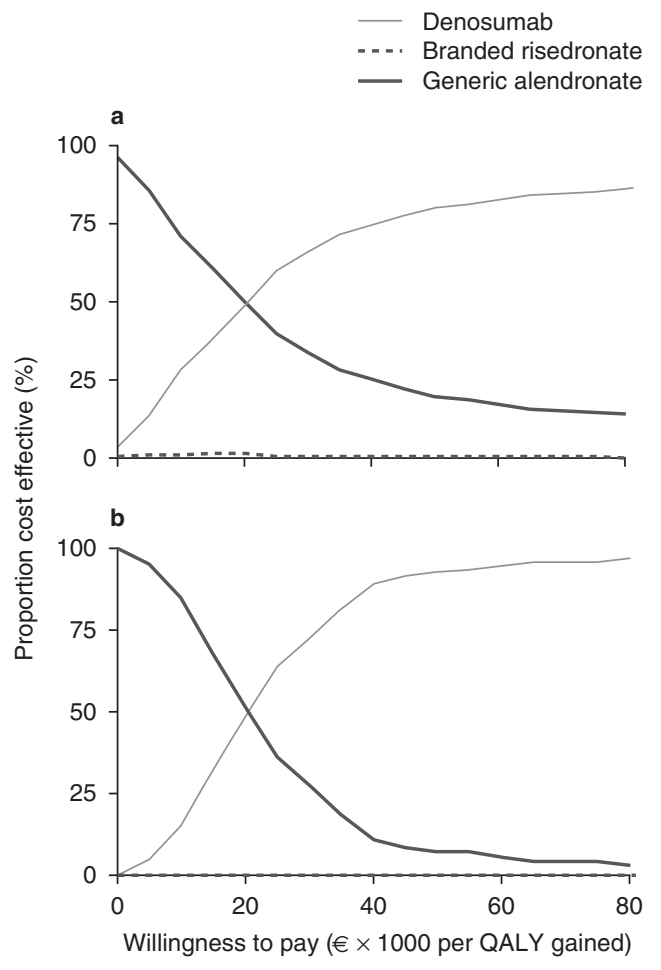

Fig. 5. Cost-effectiveness acceptability curves in women (a) with bone mineral density T-score of -2.5 or less; and (b) with prevalent vertebral fracture. Branded alendronate was not included in the graph as it was dominated by generic alendronate.

The model used in this study has been previously validated ${ }^{[20]}$ and has been used in a number of studies to model the cost effectiveness of osteoporosis management. ${ }^{[10,27,55,58,61,75,76]}$ The methodology used to incorporate compliance and persistence with oral bisphosphonates has also been recently published..$^{[10,61]}$ Nevertheless, like all models, several limitations must be taken into account. Many of these limitations (e.g. adherence data and model assumptions) have been previously extensively discussed..$^{[10,20]}$ In particular, assumptions and data on adherence to oral bisphosphonates, which play a key role in the cost effectiveness of denosumab, should be acknowledged. First, a refill gap period of 5 weeks was used to define non-persistence in the Belgian observational study. However, some patients have been identified who returned from a temporary interruption to therapy ${ }^{[77]}$ and it is difficult to include such patients in modelling because the effectiveness of oral bisphosphonates used in an interrupted way is unknown. Second, the use of a threshold of $80 \%$ for good compliance may be questionable since there is no clinically meaningful definition of good compliance. However, an MPR of $80 \%$ was commonly used to define high compliance ${ }^{[8]}$ and the use of other thresholds had limited impact on cost effectiveness in another study. ${ }^{[10]}$ In any event, the cost effectiveness of denosumab was shown to be moderately sensitive to compliance with oral bisphosphonates.

Third, assigning 3 months of costs for patients who discontinued within 6 months might not be realistic, depending on prescribing customs. Oral bisphosphonates are currently available in Belgium with a prescription for 4 or 12 weeks. The cost of a 4-week prescription was tested in a sensitivity analysis, which had limited impact on the results.

In addition, our analysis was performed from a payer's perspective rather than a societal one, in accordance with Belgian methodological guidelines for pharmacoeconomic evaluations. ${ }^{[19]}$ Taking indirect costs such as productivity losses into account would likely improve the cost effectiveness of denosumab given sensitivity analysis showed that cost effectiveness improved with higher fracture costs.

The current analyses were conducted only in a limited number of scenarios in terms of fracture risk, i.e. in women with BMD T-scores of -2.5 or less or prevalent vertebral fractures. Our model probably underestimated the cost effectiveness of denosumab in the latter group since women with a prior fracture are likely to have lower BMD than we conservatively assumed, i.e. that of the age-matched general population. Additional analyses evaluating the cost effectiveness according to absolute fracture risk would be valuable. It has been increasingly suggested that treatment should be based on absolute fracture risk rather than on BMD threshold. ${ }^{[5]}$ Recent developments in fracture risk assessment include the FRAX ${ }^{\circledR}$ tool, which estimates the 10-year probability of fracture. ${ }^{[78,79]}$ FRAX $^{\circledR}$ has recently become available in Belgium. ${ }^{[80]}$ Our simulation model is currently not able to estimate intervention thresholds.

Finally, the ability of our results to be extrapolated to other countries may be uncertain since 
fracture risk, fracture cost, drug cost and medication adherence differ between countries, and all have an impact on cost effectiveness. However, it is likely that countries with similar characteristics to those retained in our analysis will also show similar cost effectiveness. Belgium is a country with medium hip fracture $\operatorname{risk}^{[81]}$ and a prior cost-effectiveness analysis of alendronate conducted in nine European countries suggested that Belgium was in the 'medium' group, along with France and Germany. ${ }^{[54]}$ Sweden, Norway, Denmark and the UK had better cost-effectiveness ratios, while Italy and Spain had the worst estimates.

\section{Conclusion}

This cost-effectiveness analysis suggests, on the basis of currently available data, that denosumab is likely to be a cost-effective strategy compared with oral bisphosphonates (including generic alendronate) for the treatment of osteoporosis in post-menopausal women aged $\geq 60$ years in Belgium. Denosumab may therefore represent a promising treatment option to prevent osteoporotic fractures, especially given the poor adherence to oral bisphosphonates. The drug appears to have the potential to become a first-line treatment for post-menopausal women with osteoporosis. However, further studies are required to evaluate the long-term safety of, and adherence to, denosumab in real-world clinical practice, as well as head-to-head effectiveness compared with oral bisphosphonates.

\section{Acknowledgements}

Mickaël Hiligsmann and Jean-Yves Reginster contributed to the conception and design of the economic model, the analysis and interpretation of the data and the writing of the manuscript. This study was supported by an unrestricted educational grant from Amgen, which had no role in the design and conduct of the study; collection, management, analysis and interpretation of the data; and preparation of the manuscript.

The authors thank Sean Robbins, Matthew Gitlin, Enkhe Badamgarav and David Macarios of Amgen, and two anonymous reviewers for critical review and helpful comments on the manuscript. Mickaël Hiligsmann and Jean-Yves Reginster have received research grants, or consulting fees, from a number of pharmaceutical companies. The model development and validation was previously supported by an ESCEO-
Amgen Fellowship grant received at the 6th European Congress on Clinical and Economic Aspects of Osteoporosis and Osteoarthritis (Vienna, 2006).

\section{References}

1. Hiligsmann M, Bruyere O, Ethgen O, et al. Lifetime absolute risk of hip and other osteoporotic fracture in Belgian women. Bone 2008; 43 (6): 991-4

2. Kanis JA, Johnell O, Oden A, et al. Long-term risk of osteoporotic fracture in Malmo. Osteoporos Int 2000; 11 (8): 669-74

3. Johnell O, Kanis JA. An estimate of the worldwide prevalence and disability associated with osteoporotic fractures. Osteoporos Int 2006; 17 (12): 1726-33

4. Kanis JA, Johnell O. Requirements for DXA for the management of osteoporosis in Europe. Osteoporos Int 2005; 16 (3): 229-38

5. Kanis J, Burlet N, Cooper C, et al. European guidance for the diagnosis and management of osteoporosis in postmenopausal women. Osteoporos Int 2008; 19 (4): 399-428

6. Imaz I, Zegarra P, Gonzalez-Enriquez J, et al. Poor bisphosphonate adherence for treatment of osteoporosis increases fracture risk: systematic review and meta-analysis. Osteoporos Int 2010 Nov; 21 (11): 1943-51

7. Rabenda V, Hiligsmann M, Reginster JY. Poor adherence to oral bisphosphonate treatment and its consequences: a review of the evidence. Expert Opin Pharmacother 2009; 10 (14): 2303-15

8. Siris ES, Selby PL, Saag KG, et al. Impact of osteoporosis treatment adherence on fracture rates in North America and Europe. Am J Med 2009; 122 Suppl. 2: S3-13

9. Cramer JA, Amonkar MM, Hebborn A, et al. Compliance and persistence with bisphosphonate dosing regimens among women with postmenopausal osteoporosis. Curr Med Res Opin 2005; 21 (9): 1453-60

10. Hiligsmann $\mathrm{M}$, Rabenda $\mathrm{V}$, Bruyère $\mathrm{O}$, et al. The clinical and economic burden of non-adherence with osteoporosis medications. Health Policy 2010; 96: 170-7

11. Rossini M, Bianchi G, Di Munno O, et al. Determinants of adherence to osteoporosis treatment in clinical practice. Osteoporos Int 2006; 17 (6): 914-21

12. Sambrook P. Compliance with treatment in osteoporosis patients: an ongoing problem. Aust Fam Physician 2006; 35 (3): $135-7$

13. Cummings SR, Martin JS, McClung MR, et al. Denosumab for prevention of fractures in postmenopausal women with osteoporosis. N Engl J Med 2009; 361 (8): 756-65

14. European Medicines Agency. CHMP assessment report for Prolia. London: European Medicines Agency, 2009 Mar 18 [online]. Available from URL: http://www.ema.europa.eu/ docs/en_GB/document_library/EPAR__Public_assessment report/human/001120/WC500093529. $\overline{\text { pdf }}$ [Accessed 2010 July 27]

15. Kendler DL, Bessette L, Hill CD, et al. Preference and satisfaction with a 6-month subcutaneous injection versus a weekly tablet for treatment of low bone mass. Osteoporos Int 2010; 21 (5): 837-46

16. Claxton AJ, Cramer J, Pierce C. A systematic review of the associations between dose regimens and medication compliance. Clin Ther 2001; 23 (8): 1296-310 
17. Briggs A, Claxton K, Sculpher M. Decision modelling for health economic evaluation. 2nd ed. New York: Oxford University Press, 2007

18. Drummond M, Sculpher M, O'Brien B, et al. Methods for the economic evaluation of health care programmes. 3rd ed. New York: Oxford University Press, 2007

19. Cleemput I, van Wilder P, Huybrechts M, et al. Belgian methodological guidelines for pharmacoeconomic evaluations: toward standardization of drug reimbursement requests. Value Health 2009; 12 (4): 441-9

20. Hiligsmann M, Ethgen O, Bruyere O, et al. Development and validation of a Markov microsimulation model for the economic evaluation of treatments in osteoporosis. Value Health 2009; 12 (5): 687-96

21. Dere W, Avouac B, Boers M, et al. Recommendations for the health economics analysis to be performed with a drug to be registered in prevention or treatment of osteoporosis. Calcif Tissue Int 1998; 63 (2): 93-7

22. Reginster JY, Gillet P, Ben Sedrine W, et al. Direct costs of hip fractures in patients over 60 years of age in Belgium. Pharmacoeconomics 1999; 15 (5): 507-14

23. Autier P, Haentjens $\mathrm{P}$, Bentin J, et al. Costs induced by hip fractures: a prospective controlled study in Belgium. Belgian Hip Fracture Study Group. Osteoporos Int 2000; 11 (5): 373-80

24. Gabriel SE, Tosteson AN, Leibson CL, et al. Direct medical costs attributable to osteoporotic fractures. Osteoporos Int 2002; 13 (4): 323-30

25. Melton 3rd LJ, Gabriel SE, Crowson CS, et al. Costequivalence of different osteoporotic fractures. Osteoporos Int 2003; 14 (5): 383-8

26. Bouee S, Lafuma A, Fagnani F, et al. Estimation of direct unit costs associated with non-vertebral osteoporotic fractures in five European countries. Rheumatol Int 2006; 26 (12): 1063-72

27. Hiligsmann M, Ethgen O, Bruyere O, et al. An economic evaluation of quantitative ultrasonometry as pre-screening test for the identification of patients with osteoporosis. Dis Manage Health Outcomes 2008; 16: 429-38

28. Hiligsmann M, Ethgen O, Richy $F$, et al. Utility values associated with osteoporotic fracture: a systematic review of the literature. Calcif Tissue Int 2008; 82 (4): 288-92

29. Kanis JA, Oden A, Johnell O, et al. The burden of osteoporotic fractures: a method for setting intervention thresholds. Osteoporos Int 2001; 12 (5): 417-27

30. Klotzbuecher CM, Ross PD, Landsman PB, et al. Patients with prior fractures have an increased risk of future fractures: a summary of the literature and statistical synthesis. J Bone Miner Res 2000; 15 (4): 721-39

31. Marshall D, Johnell O, Wedel H. Meta-analysis of how well measures of bone mineral density predict occurrence of osteoporotic fractures. BMJ 1996; 312 (7041): 1254-9

32. Kanis JA, Johnell O, Oden A, et al. Risk of hip fracture according to the World Health Organization criteria for osteopenia and osteoporosis. Bone 2000; 27 (5): 585-90

33. Looker AC, Wahner HW, Dunn WL, et al. Updated data on proximal femur bone mineral levels of US adults. Osteoporos Int 1998; 8 (5): 468-89

34. Boonen S, Kaufman JM, Reginster JY, et al. Patient assessment using standardized bone mineral density values and a national reference database: implementing uniform thresholds for the reimbursement of osteoporosis treatments in Belgium. Osteoporos Int 2003; 14 (2): 110-5

35. Johnell O, Kanis JA, Oden A, et al. Predictive value of BMD for hip and other fractures. J Bone Miner Res 2005; 20 (7): 1185-94

36. Kanis JA, Johnell O, De Laet C, et al. A meta-analysis of previous fracture and subsequent fracture risk. Bone 2004; 35 (2): $375-82$

37. Johnell O, Kanis JA, Oden A, et al. Fracture risk following an osteoporotic fracture. Osteoporos Int 2004; 15 (3): 175-9

38. National Institute of Statistics. Directorate-General Statistics and Economic Information. Mortality tables 2004 and 2002-2004. Brussels: National Institute of Statistics, 2008

39. Oden A, Dawson A, Dere W, et al. Lifetime risk of hip fractures is underestimated. Osteoporos Int 1998; 8 (6): 599-603

40. Johnell O, Kanis JA, Oden A, et al. Mortality after osteoporotic fractures. Osteoporos Int 2004; 15 (1): 38-42

41. Cauley JA, Thompson DE, Ensrud KC, et al. Risk of mortality following clinical fractures. Osteoporos Int 2000; 11 (7): 556-61

42. Kanis JA, Oden A, Johnell O, et al. Excess mortality after hospitalisation for vertebral fracture. Osteoporos Int 2004; 15 (2): 108-12

43. Kanis JA, Oden A, Johnell O, et al. The components of excess mortality after hip fracture. Bone 2003; 32 (5): 468-73

44. Lips P, van Schoor NM. Quality of life in patients with osteoporosis. Osteoporos Int 2005; 16 (5): 447-55

45. Silverman SL, Minshall ME, Shen W, et al. The relationship of health-related quality of life to prevalent and incident vertebral fractures in postmenopausal women with osteoporosis: results from the Multiple Outcomes of Raloxifene Evaluation Study. Arthritis Rheum 2001; 44 (11): 2611-9

46. Tosteson AN, Hammond CS. Quality-of-life assessment in osteoporosis: health-status and preference-based measures. Pharmacoeconomics 2002; 20 (5): 289-303

47. Belgian Federal Government. FPS economy, SMEs, independent professions and energy: consumer price indexes. 2009 [online]. Available from URL: http://economie.fgov. be/fr/statistiques/chiffres/economie/prix_consommation/in dices_prix_consommation/index.jsp [Accessed 2010 Mar 1]

48. National Institute for Health and Clinical Excellence. Systematic reviews of clinical effectiveness prepared for the guideline 'Osteoporosis: assessment of fracture risk and the prevention of osteoporotic fractures in individuals at high risk'. London: NICE, 2008 Sep [online]. Available from URL: http://www.nice.org.uk/nicemedia/live/11621/42362/ 42362.pdf [Accessed 2010 Mar 1]

49. Rabenda V, Mertens R, Fabri V, et al. Adherence to bisphosphonates therapy and hip fracture risk in osteoporotic women. Osteoporos Int 2008; 19 (6): 811-8

50. Huybrechts KF, Ishak KJ, Caro JJ. Assessment of compliance with osteoporosis treatment and its consequences in a managed care population. Bone 2006; 38 (6): 922-8

51. Belgian Center for Pharmacotherapeutic Information [online]. Available from URL: http://www.cbip.be/GGR/ MPG/MPG_NI.cfm\#MP_04090 [Accessed 2010 Apr 1]

52. Greenspan SL, Emkey RD, Bone HG, et al. Significant differential effects of alendronate, estrogen, or combination therapy on the rate of bone loss after discontinuation of treatment of postmenopausal osteoporosis: a randomized, 
double-blind, placebo-controlled trial. Ann Intern Med 2002; 137 (11): 875-83

53. Stock JL, Bell NH, Chesnut 3rd CH, et al. Increments in bone mineral density of the lumbar spine and hip and suppression of bone turnover are maintained after discontinuation of alendronate in postmenopausal women. Am J Med 1997; 103 (4): 291-7

54. Strom O, Borgstrom F, Sen SS, et al. Cost-effectiveness of alendronate in the treatment of postmenopausal women in 9 European countries: an economic evaluation based on the fracture intervention trial. Osteoporos Int 2007; 18 (8): 1047-61

55. Hiligsmann M, Reginster JY. Potential cost-effectiveness of denosumab for the treatment of postmenopausal osteoporotic women. Bone 2010; 47 (1): 34-40

56. Bone HG, Bolognese MA, Yuen CK, et al. Effects of denosumab treatment and discontinuation on bone mineral density and bone turnover markers in postmenopausal women with low bone mass. J Clin Endocrinol Metab 2011; 96 (4): 972-80

57. Lekkerkerker F, Kanis JA, Alsayed N, et al. Adherence to treatment of osteoporosis: a need for study. Osteoporos Int 2007; 18 (10): 1311-7

58. Hiligsmann M, Rabenda V, Gathon HJ, et al. Potential clinical and economic impact of nonadherence with osteoporosis medications. Calcif Tissue Int 2010; 86 (3): 202-10

59. Strom O, Borgstrom F, Kanis JA, et al. Incorporating adherence into health economic modelling of osteoporosis. Osteoporos Int 2009; 20 (1): 23-34

60. Cramer JA, Roy A, Burrell A, et al. Medication compliance and persistence: terminology and definitions. Value Health 2008; 11 (1): 44-7

61. Hiligsmann M, Gathon HJ, Bruyere O, et al. Cost-effectiveness of osteoporosis screening followed by treatment: the impact of medication adherence. Value Health 2010; 13 (4): 394-401

62. Strom O, Landfeldt E, Robbins S, et al. Adherence to treatment of osteoporosis and fracture risk: the Swedish adherence register analysis (SARA) [abstract no. P109]. Osteoporos Int 2010; 21 Suppl. 1: S29

63. Cramer JA, Gold DT, Silverman SL, et al. A systematic review of persistence and compliance with bisphosphonates for osteoporosis. Osteoporos Int 2007; 18 (8): 1023-31

64. Kendler DL, McClung MR, Freemantle N, et al. on behalf of the DAPS Investigators. Adherence, preference, and satisfaction of postmenopausal women taking denosumab and alendronate. Osteoporos Int. Epub 2010 Sep 9

65. Lewiecki EM. Is denosumab better than alendronate in the treatment of osteoporosis? Nat Clin Pract Rheumatol 2009; 5 (2): $72-3$

66. Brown JP, Prince RL, Deal C, et al. Comparison of the effect of denosumab and alendronate on bone mineral density and biochemical markers of bone turnover in postmenopausal women with low bone mass: a randomized, blinded, phase 3 trial. J Bone Miner Res 2009; 14: 1-34

67. Cleemput I, Neyt M, Thiry N, et al. Valeurs seuils pour le rapport coût-efficacité en soins de santé. Health Technology Assessment (HTA). Bruxelles: centre fédéral d'ex- pertise des soins de santé (KCE), 2008. KCE Reports 100B (D/2008/10.273/95)

68. Raftery J. NICE: faster access to modern treatments? Analysis of guidance on health technologies. BMJ 2001; 323: 1300-3

69. Tosteson AM, Malton LJ, Dawson-Hughes B, et al. National Osteoporosis Foundation Guide Committee. Costeffective osteoporosis treatment thresholds: the United States perspective. Osteoporos Int 2008; 4: 437-47

70. Cranney A, Guyatt G, Griffith L, et al. Meta-analyses of therapies for postmenopausal osteoporosis: IX. Summary of meta-analyses of therapies for postmenopausal osteoporosis. Endocr Rev 2002; 23 (4): 570-8

71. Ringe JD, Moller G. Differences in persistence, safety and efficacy of generic and original branded once weekly bisphosphonates in patients with postmenopausal osteoporosis: 1-year results of a retrospective patient chart review analysis. Rheumatol Int 2009; 30 (2): 213-21

72. Sheehy O, Kindundu CM, Barbeau M, et al. Differences in persistence among different weekly oral bisphosphonate medications. Osteoporos Int 2009; 20 (8): 1369-76

73. Bobba RS, Beattie K, Parkinson B, et al. Tolerability of different dosing regimens of bisphosphonates for the treatment of osteoporosis and malignant bone disease. Drug Saf 2006; 29 (12): 1133-52

74. Borgstrom F, Johnell O, Kanis JA, et al. Cost effectiveness of raloxifene in the treatment of osteoporosis in Sweden: an economic evaluation based on the MORE study. Pharmacoeconomics 2004; 22 (17): 1153-65

75. Hiligsmann M, Bruyere O, Reginster JY. Cost-effectiveness of strontium ranelate versus risedronate in the treatment of postmenopausal osteoporotic women aged over 75 years. Bone 2010; 46 (2): 440-6

76. Hiligsmann M, Bruyere O, Reginster JY. Cost-utility of long-term strontium ranelate treatment for postmenopausal osteoporotic women. Osteoporos Int 2010; 21 (1): 157-65

77. Brookhart MA, Avorn J, Katz JN, et al. Gaps in treatment among users of osteoporosis medications: the dynamics of noncompliance. Am J Med 2007; 120 (3): 251-6

78. Kanis JA, Johnell O, Oden A, et al. FRAX and the assessment of fracture probability in men and women from the UK. Osteoporos Int 2008; 19 (4): 385-97

79. Kanis JA, Oden A, Johansson H, et al. FRAX and its applications to clinical practice. Bone 2009; 44 (5): 734-43

80. Johansson H, Kanis JA, McCloskey EV, et al. A FRAX ${ }^{\circledR}$ model for the assessment of fracture probability in Belgium. Osteoporos Int 2011 Feb; 22 (2): 453-61

81. Kanis JA, Johnell O, De Laet C, et al. International variations in hip fracture probabilities: implications for risk assessment. J Bone Miner Res 2002; 17 (7): 1237-44

Correspondence: Dr Mickaël Hiligsmann, Pharmacoeconomics Research Unit, HEC-ULg Management School, University of Liège, Boulevard du Rectorat 7, Bât. B31, 4000 Liège, Belgium.

E-mail: m.hiligsmann@ulg.ac.be 